\title{
NETWORKS AND TECHNOLOGIES
}

We are moving away from a business world of "me, myself, and I". Individuals, and also individual companies, are not able to create the very complex innovative and carbon-neutral products and services that are needed in the 21 st century. More and more people and companies will be collaborating and combining resources and capabilities in inter-personal and inter-organizational networks. The value lies not so much in the individual or in the company: the network is where the value lies. Eric van Heck, professor at Erasmus University's Rotterdam School of Management, chose five books that have inspired his research in the last couple of years. Each of the books analyses and explains the role and accelerating impact of technology. The books discuss the necessary - but not always sufficient - conditions for using technologies successfully.
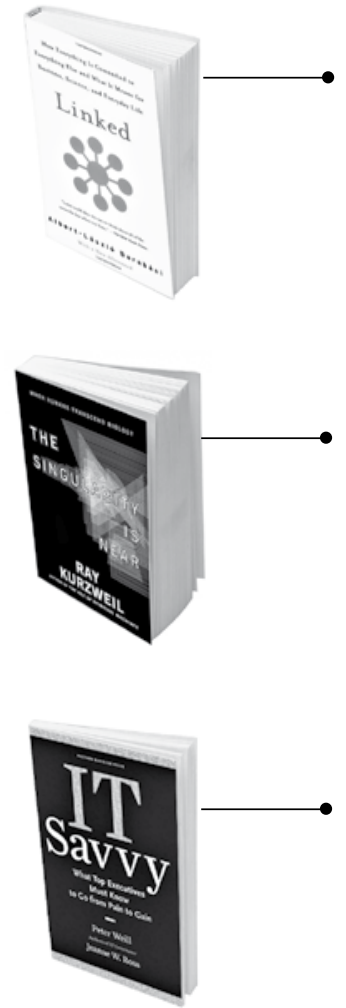

IT SAVVY. What top executives must know to go from pain to gain. Peter Weill and Jeanne Ross. Boston, MA: Harvard Business Press, 2009. 182 p.

During my time as a visiting scholar at MIT Sloan Center for Information Systems Research (CISR), I learned about CISR's research and its profound impact. The IT Savvy book is a great summary of their work and is written for non-IT executives. IT Savvy explains in great detail how companies gain maximum value from information technology. It explains the role of the digitized platform for companies to develop business agility and introduce business innovation.

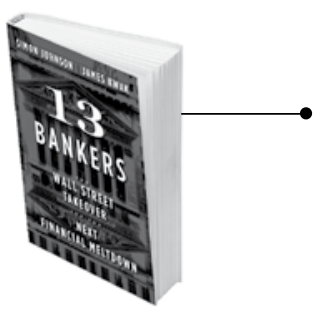

13 BANKERS: The Wall Street takeover and the next financial meltdown. Simon Johnson and James Kwak. New York: Pantheon, 2010. 320 p.

This book explains the takeover by Wall Street oligarchs (such as Bank of America, Citibank, Goldman Sachs, J.P. Morgan and Wells Fargo) of crucial parts of the economy and the government. The book shows the power of formal and informal personal networks at Wall Street and its regulatory bodies, but also the greedy bonus culture of some bankers. They argue for the limitation of power of banks by downsizing and introducing much stricter regulations to save society the burden of the next financial meltdown.

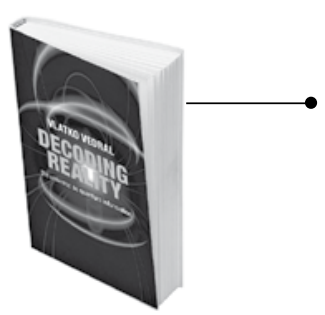

DECODING REALITY: The universe as Quantum information. Vlatko Vedral. Oxford: Oxford University Press, 2010. $256 \mathrm{p}$.

This book argues that information is far more fundamental than matter or energy because it can successfully be applied to macroscopic interactions, such as economic and social phenomena, and also microscopic interactions such as energy and matter. Information is defined as a measure of how surprising something is, low probability events contain a high degree of information. Provoking book that presents an advanced information theory. 